\title{
A Dynamic Control System for Adjusting Prices and Quality of Service in DS Enabled Networks
}

\author{
Kimmo Pulakka \\ Tampere University of Technology, Institute of Communications Engineering
}

\begin{abstract}
In the differentiated services (DS) enabled networks, Internet service providers (ISPs) establish service level agreements (SLAs) with their customers. Prices of the SLAs and quality of data transmission services should correspond to the requirements of the customers and operations aims of the ISPs. For this reason, IP domains of the ISPs should contain a control system for setting the selling prices of the SLAs and DS services used for data flows of the SLAs. The control system should consider dynamically both requirements of the customers and quality of end-to-end data transmission services. This task is complex because the customers and operators of other networks may not give useful information to the ISPs for the control task. For this reason, a new fuzzy controller based control system is developed. The system operates using local measurements of ingress routers of IP domains about the behaviour of the customers. It does not inquire the level of satisfaction of the customers about offered data transmission services, or capacities of networks of other operators. The operations of the control system are tested using the Network Simulator 2.
\end{abstract}

Key words: Multi-service Internet, Differentiated Services, Service Level Agreements, Fuzzy controllers

\section{INTRODUCTION}

In the differentiated services (DS) model [1] defined by the IETF (Internet Engineering Task Force), Internet service providers (ISPs) establish service level agreements (SLAs) with their customers. These SLAs define transmission costs of the customers and expected quality of the offered data transmission services [2]. The ISPs are responsible for their customers for

The original version of this chapter was revised: The copyright line was incorrect. This has been corrected. The Erratum to this chapter is available at DOI: 10.1007/978-0-387-35620-4_43 
ensuring that the offered end-to-end data transmission services correspond to the quality of service definitions of established SLAs at least in some statistical level. For this reason, the ingress routers of IP domains of the ISPs should be capable of estimating quality of end-to-end data transmission services.

In long term, the ISPs are certainly able to control quality of the data transmission services of their own networks by dimensioning capacities of components of their networks [3], planning the characteristics of PHBs (PerHop-Behaviours) of the routers and performing traditional traffic control functions on the edges of their networks. However, estimation of quality of end-to-end data transmission services is a more complex task, if data packets of transferred data flows are routed through IP domains of several network operators. Firstly, the DS model does not provide any kinds of data flowbased control or resource reservation functions through all networks used for transferring of data packets. Secondly, network operators are not willing to share all information about resources of their networks between other operators [4].

In this situation, some research groups have studied sharing of information between IP domains of different network operators and cooperation of bandwidth brokers (BBs) of different IP domains (e.g. [4], [5]) for ensuring quality of end-to-end data transmission services. Another way to be aware of quality of end-to-end data transmission services is simply monitor quality of offered data transmission services [6][7]. Different techniques can be used for this purpose. Monitoring results have already used for different traffic control functions (e.g. [8]). From perspective of network operators, fruition of their economical profit aims is obviously an important issue. For this reason, several research groups have studied the relationship of selling prices and quality of data transmission services (e.g. [9], [2]).

In this paper, we describe a control system for IP domains of ISPs. The control system sets prices and DS services of the SLAs according to operation targets of the ISPs. The control system is designed for a future network environment, where customers are able to select their ISPs and SLAs dynamically and freely irrespective of their access network operators. In addition, it can also be used for controlling the SLAs if ISP selection of a customer would be static and the customer of a particular ISP would just select among the SLAs of that ISP for his data transmission needs.

The principal advantage of the control system is independence of the control operations. The system can consider opinions of both operators and customers about suitable prices and quality of data transmission services, although it does not monitor quality of data transmission services frequently or inquire opinions of the customers. The operation targets of the ISPs are 
described using manually tuned mamdani type of fuzzy controllers. The well-known advantage of this type of controllers is that system experts (network operators) can affect control operations of controllers easily by tuning the controllers according to their heuristical control aims [10]. The fuzzy controllers have already been used for different control tasks of different networks in several studies (e.g. [11], [12], [13]).

The network environment and the principles of the developed control system are described in Section 2. In Section 3, the mechanism is tested using Network Simulator 2 [14]. Finally, some conclusions are given.

\section{THE CONTROL SYSTEM}

\subsection{Description of the studied network environment}

The developed control system is designed for a network environment where several interconnected DS enabled IP networks offer multiple data transmission services. Terminals of the customers are connected to the IP network area via access networks. The access networks are broadband networks, which are able to transfer IP packets. Data flows of the customers flow through the (source) access networks, IP domains of the ISPs of customers, backbone networks, IP domains of ISPs of receivers, and (destination) access networks to receiving terminals.

It is assumed that the ISPs define several alternative service level agreements for both real-time and non-real-time data transmissions. The agreements are defined by the quality of service factors and price. The quality of service factors are 1) the minimum data transmission rate, 2) the maximum packet loss ratio, 3) the maximum delay and 4) the maximum delay variation. The price of the SLA defines the cost of the customers per data unit, if they establish agreement with ISPs for sending data. From this point of view, we expect that senders of the data flows are paying for their data transmissions on a per-packet or per-byte basis.

The ISPs advertise their SLAs for potential customers. For this operation, we expect that the source terminals have some mechanism for inquiring information about alternative SLAs of the ISPs or the ISPs can dynamically send this information to the source terminals. In this way, the customers have always information about SLAs of competing ISPs. They know prices of the SLAs and advertised quality of end-to-end data transmission services, which should be reached using the SLAs.

At the beginning of transmission of data flows, the customers firstly select the suitable SLAs for their transmissions. The terminals of the customers inform the ISPs about the SLA selections by copying an index of 
the selected SLAs into the DS label of transferred data packets. When an ingress router receives a data packet, it first reads an indication of the selected SLA from the DS label of the IP packet. After this, it checks the valid DS codepoint of the SLA, copies the index of the DS codepoint into DS label of the IP packet and forwards the packet to the IP domain.

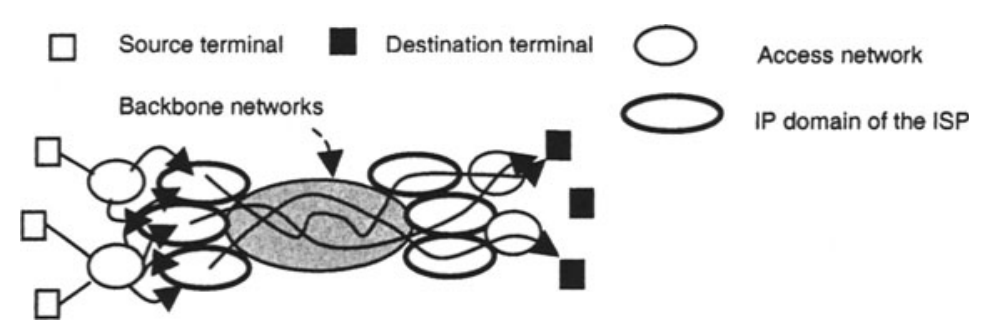

Figure 1. Layout of the studied network environment

Every ingress router includes a control system for defining prices and DS services of defined SLAs. The ingress routers do not contain any admission control functions. Data flows of all customers who are willing to accept some of the offered service level agreements with the ISPs are transferred. For control and billing operations, it is expected that ingress routers store information about number of customers, SLA selections of the customers and amount of transferred data of the SLAs.

\subsection{Components of the control system}

Principal elements of the control system are a state-chart of the control solutions and a fuzzy controller. The state-chart describes alternative combinations of prices and DS codepoints, which can be set for the SLAs. The fuzzy controller defines suitability values of different combinations of the prices and the DS services (states of the chart) for the controlled SLAs. The manually tuned Mamdani type of fuzzy controller [11] is used for this purpose.

Every state of the chart includes three characteristic factors. These factors are: 1) codepoint, 2) selling price and 3) using cost. 'Codepoint' values simply indicate the DS services, which can be used for transferring data flows of customers through IP domains of ISPs. 'Selling price' values indicate selling prices of the SLAs (EUR/data unit), if the DS service (codepoint) of the state is used for transferring data of the customers. Correspondingly, the 'using cost' values indicate costs of the ISPs (EUR/data unit), if the DS service (codepoint) of the state is used for transferring data of the customers. 
The states of the chart contain also three 'behavior' databases for storing information about behavior of the customers. The first database stores information about number of customers using the defined SLAs, if the 'selling price' of the state is used as the price of the SLAs and the DS service of the state is used for transferring data flows of the customers. The second database stores information about amount of transferred data of the customers in the above-described situation. The third database ('the moving database') stores changes of number of customers and amount of transferred data of the SLAs, if a combination of prices and DS services of the SLAs is changed to classified directions on the state-chart. The classified directions are: 1) up, 2) up-right, 3) right, 4) down-right, 5) down 6) down-left, 7) left, and 8) up-left. Structure of the state-chart of the control system is described in Fig 2.

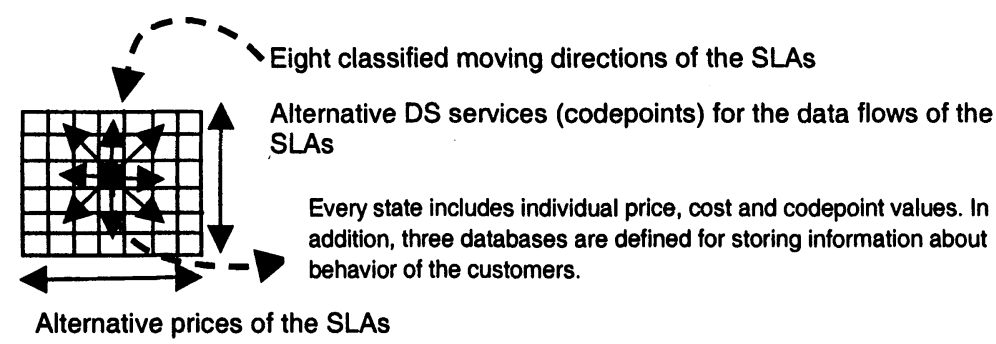

Figure 2. Structure of the state-chart of the control system

The fuzzy controller contains two input variables and one output variable. The input variables are: 1) customer ratio, and 2) profit ratio. The output variable of the controller simple indicates how suitable the alternative state of the state-chart is for the controlled SLA from ISPs point of views. A numeric value range of the output variable is $[0,1]$. Suitability of alternative states increase, when numeric value of the output variable increase. Calculation of numeric values of the input variables is described in equations 1-2.

Customer ratio $=\frac{\text { Avg. \# of customers(SLA,state) }}{\text { Expected avg. \# of customers(SLA) }}$

where:

SLA = Currently controlled SLA

state = Alternative state of the state - chart 


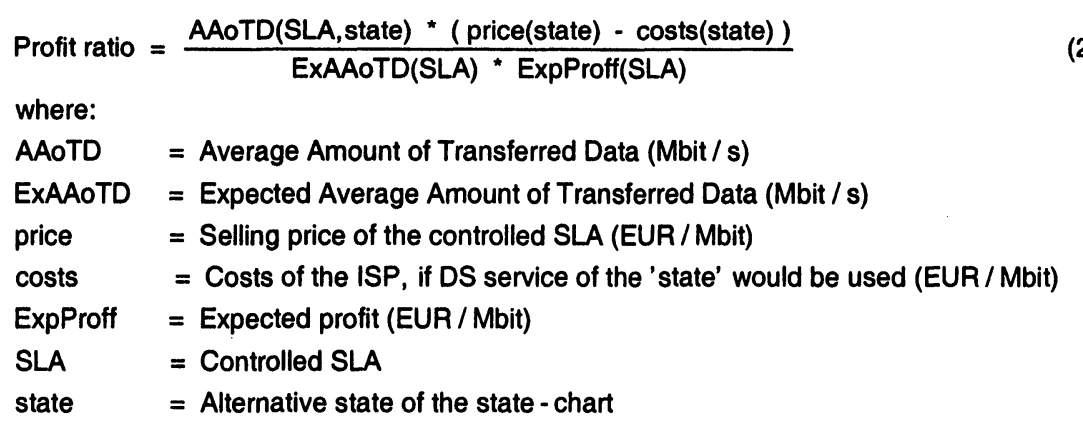

The 'customer ratio' input variable compares expected and estimated average number of customers using the controlled SLA, if the alternative state of the state-chart would be selected for the controlled SLA. Correspondingly, the 'profit ratio' input variable compares expected and estimated economical profit of the ISP (EUR/s) by selling the controlled SLA in the above-described situation.

The ISPs tune manually the linguistic variables and rule bases of the fuzzy controller according to their control aims. Manual tuning of the fuzzy controller enables the ISPs to consider how suitable different states of the state-chart are for the controlled SLA. The input variables of the controller indicate how consequences of state selections would correspond with their control aims. Correspondingly, the rule base of the controller defines how suitable different consequences are from the ISP point of view.

\subsection{Operations of the control system}

The control system includes the initialisation and the using phases. In the initialisation phase, ISPs initialise the state-chart, the first prices and DS services of the SLAs, and the fuzzy controller. After the initialisation phase, the control system is ready for use. It controls the SLAs periodically. In practice, the control system changes states of the SLAs on the state-chart. The current locations of the SLAs define the selling prices and the DS services of the SLAs. The price and the DS service selections of the SLAs are constant between sequential control moments. Locations of the SLAs are defined using the special control procedure. The control procedure includes seven phases. In the operational order, these phases are: 1) storing information about behaviour of the customers into the databases, 2) randomisation of the next controlled SLA, 3) definition of alternative states, 4) calculation of suitability values of the alternative states, 5) selection of price and DS service for the controlled SLAs, 6) updating advertise 
information of the controlled SLA, and 7) calculation of the next control moment.

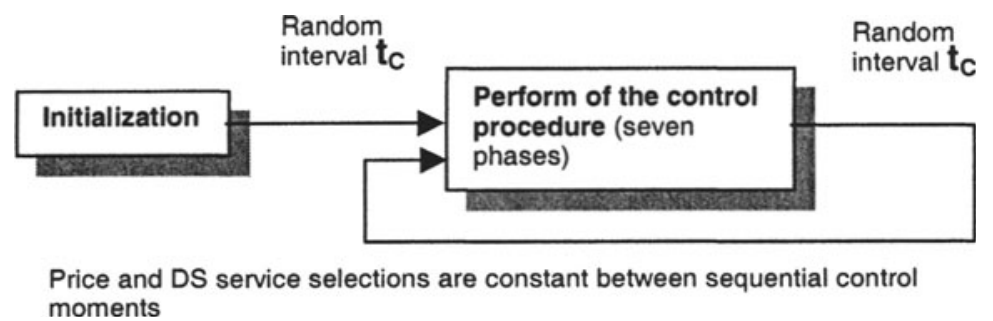

Figure 3. Operations of the control system

In the first phase, the control system calculates how the previous control decisions have affected use of the controlled SLA. Firstly, the control system measures how many customers have used the SLA and how much data has transferred using the SLA during the previous control interval. These measurements with the measurement time are stored into the responsible 'behaviour' databases of the current state of the SLA on the state-chart. Secondly, the control system calculates differences of number of customers and amount of transferred data between the recent measurements and the last measurements on the previous state of the SLA. Results of these calculations with the calculation time and the moving direction of the SLA are stored into the 'moving database' of the last state of the SLA. The moving direction indicates to what classified direction the state of the SLA has changed from the last state. The control operations of the phase are described in Fig. 4.

In the 'randomisation of the next controlled SLA' phase, the control system randomises the SLA, whose price and DS service are adjusted at next. During a control moment, only one SLA is controlled. In this way, it is easier to measure how the control actions affect behaviour of the customers. The controlled SLA is randomised using the uniform distribution.

In the 'definition of alternative states' phase, the control system defines the alternative states of the state-chart, which could be selected for the controlled SLA. From a current state of the SLA, the state of the SLA can be changed to the eight classified moving directions. Number of alternative states in each direction changes according to the definitions of the ISPs.

During the 'calculation of suitability values of the alternative states' phase, the control system calculates suitability values for every alternative state. If the 'behaviour' databases of the alternative states include enough information for the fuzzy controller, the fuzzy controller is used for calculating suitability values of the states. Otherwise, suitability values of the alternative states are randomised from the uniform distribution. In the 
'selection of price and DS service for the controlled SLAs' phase, the state whose suitability value is the largest, is selected for the next state of the controlled SLA on the state-chart.

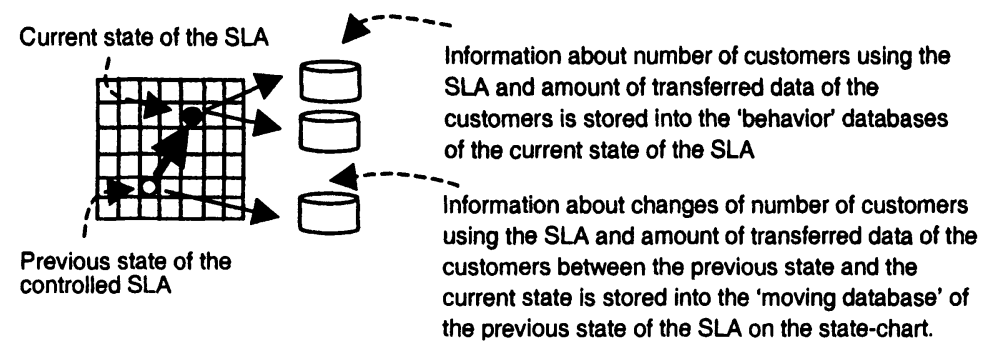

Figure 4. Monitoring of behaviour of the customers

In the 'updating advertise information of the controlled SLA' phase, price and quality of service information of the controlled SLA is updated for an advertisement of the SLA. For estimating quality of end-to-end data transmission services, it is expected that the ISPs have some traffic monitoring systems. These systems are able to estimate quality of end-to-end data transmission services in some probabilistic level, if the valid DS service of the SLA is used for transferring data flows through IP domains of the ISPs. In this study, we do not define what methods the ISPs should use for the estimation task. In the 'calculation of the next control moment' phase, the next control moment of the SLAs of the ISP is randomised from the uniform distribution. The control intervals vary on the range $\left[t_{C}, 2 * t_{C}\right]$, where the ISP defines a value of the factor $t_{C}$.

\section{SIMULATIONS}

\subsection{Simulated network environment}

We tested the operations of the control system using the Network Simulator 2 (NS2). The principal aim of the test was to verify the basic operations of the system. The environment consisted of 80 source and destination terminal pairs, 80 access routers representing the access networks of the source terminals, five DS edge routers for five different ISPs, one DS core router, a destination DS edge router and one access router for the destination terminals. Capacities of the transmission links between the routers were defined so that the link between the core and destination DS 
edge routers was the bottleneck router. The simulated network environment is described in Fig. 5.

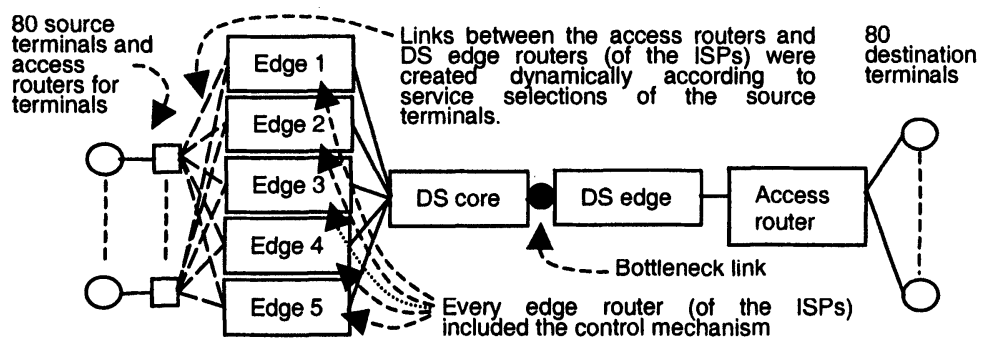

Figure 5. Simulated network environment

The queue of the bottleneck link included two physical queues, which consisted of three virtual queues. In this way, the DS core router included six DS services handled by six codepoint values. The first physical queue was used for the data flows of real-time applications, while the second physical queue was used for the traffic of non-real-time applications. The operational weights of the physical queues were selected so that most of transmission capacity of the bottleneck link was allocated for real-time data flows.

Every ISP reserved one SLA for data flows of real-time applications and two SLAs for non-real-time traffic. The control system of the edge routers selected real-time DS services of the core router for the real-time SLA and non-real-time DS services for non-real-time SLAs. Costs of using different DS services of the core router were different, but equal for every ISP. Operation aims of five defined ISPs varied. Expected average numbers of customers of ISPs $1-5$ were $25,25,60,30$ and 5 customers. Correspondingly, expected average economical profits of ISPs 1-5 were 29.7, 29.7, 148.7, 29.7 and 0.6 monetary unit/s. Tuning principles of the fuzzy controllers of the ISPs also varied. The fuzzy controllers of ISPs 1-3 calculated the largest suitability values for the states, which produced maximum profits for the ISPs and a good service for the customers. The fuzzy controllers of ISPs 4-5 calculated large suitability values for the states, which should ensure a good service for the customers with just an expected economical profit of the ISPs.

Non-real-time SLAs were used for data flows of the FTP application. Data of the FTP application was transferred using the TCP protocol. Length of transferred files varied randomly. The exponential distributions were used to define the lengths idle periods between sequential data transmissions. Real-time SLAs were used for data flows of the CBR application. Data of the CBR application was transferred using the UDP protocol. The 
exponential distributions were used to define the lengths of the active and idle periods of the data transmissions of the CBR application of the source user terminals.

\subsection{The control results}

The total simulation time was 4500 seconds. Price and DS service selections of the ISPs and behaviour of the customers were observed during the test. The control targets were consciously set so that the targets of all ISPs were impossible to be fulfilled. In this situation, the control systems tried to reach the best possible control results. The average control results of all three SLAs of the ISPs are described in Table 1.

Table 1. The average simulation results

\begin{tabular}{llllll}
\hline ISP \# & $\begin{array}{l}\text { Avg. DS } \\
\text { service } \\
\text { [best,worst] } \\
\rightarrow[11.5,13.5]\end{array}$ & $\begin{array}{l}\text { Avg. price } \\
\text { (monetary } \\
\text { unit/Mbit) }\end{array}$ & Price / Costs & $\begin{array}{l}\text { Average } \\
\text { profit / } \\
\text { expected } \\
\text { profit } \\
\text { (monetary } \\
\text { unit/s) }\end{array}$ & $\begin{array}{l}\text { Customer } \\
\text { ratio / } \\
\text { percentage of } \\
\text { all transferred } \\
\text { data }\end{array}$ \\
& & & & $63.63 / 29.7$ & $0.25 / 16.97$ \\
\hline 1 & 12.46 & 23.29 & 12.35 & $61.28 / 29.7$ & $0.27 / 20.93$ \\
2 & 12.78 & 22.05 & 11.35 & $51.28 / 148.7$ & $0.11 / 18.27$ \\
3 & 12.63 & 18.61 & 11.51 & $27.96 / 29.7$ & $0.24 / 21.95$ \\
4 & 12.40 & 14.53 & 8.42 & $16.04 / 0.6$ & $1.31 / 21.88$ \\
\hline 5 & 12.22 & 10.26 & 3.14 & & \\
\hline
\end{tabular}

The control targets of the ISPs one, two and four are fulfilled most optimally. The profit values of ISPs 1-2 were significantly larger than the expectations. This was also the control target of ISPs 1-2. The economical profit of ISP 4 corresponded quite optimally to the expectation of the ISP. ISP 4 could offer better data transmission services in lower price for the customers than ISPs 1-2 because the fuzzy controller of ISP 4 did not try to reach better profit than the expectation of the ISP as the fuzzy controllers of ISPs 1-2 did. The average profit of ISP 3 did not correspond to expectation of the ISP. The desired profit of ISP 3 was impossible to reach in the simulated network environment, because the profit expectations of the other ISPs were significantly smaller. The average profit of ISP 5 was clearly the smallest. The reason for this result was the small profit expectation of the ISP. Number of customers corresponded also to the expectation of the ISP. However, the economical profit was significantly larger than the expectation of the ISP. The control system operated long period, before it found the correct combinations of prices and DS services for the 'real-time' SLA of the ISP (see Fig. 6). Another obvious reason for the too large profit is the extremely small profit target of the ISP. The state-chart of the control system 
included too few alternative price values for setting prices of the SLAs carefully enough according to the profit target of the ISP.

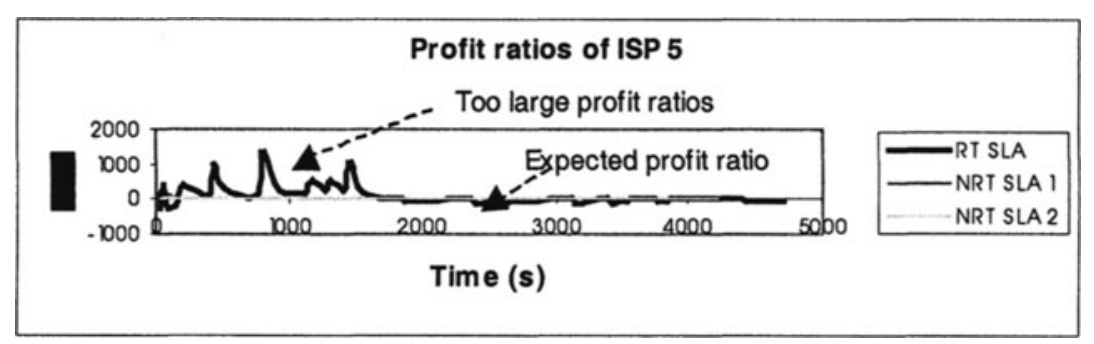

Figure 6. Profit ratios of ISP 5

\section{CONCLUSIONS}

In this paper, a new control system for setting prices and DS services of the SLAs of the ISPs is defined. The control system is designed for a future network environment, where customers are able to select their ISPs and SLAs dynamically and freely irrespective of their access network operators. The principal advantage of the control system is the capability of considering the opinions of both operators and customers about suitable prices and quality of data transmission services, without monitoring quality of data transmission services frequently or inquiring opinions of the customers.

Operations of the control system were verified by a simulation test. According to the simulation results, the control system could control SLAs of the ISPs, although the control targets of the ISPs varied heavily. The control actions of the control system correspond more strictly to the control aims of the ISPs, if the aims are possible to be fulfilled in the network environment.

In the test, behavior of the control system of ISP 5 also indicates that the 'learning speed' of the control system varies randomly. The control system may need long period for finding the optimal control decisions, if the first random price and DS service selections are very incorrect compared to the control targets. Finally, it is important to remember that the operation speed of the system depends on the behaviours of the customers. Behaviour of the customers gives the needed information for the control actions. From this point of view, the control system cannot operate faster than the customers establish SLAs. However, the control system does not require any specific control frequence. It can be adapted to different SLA establishing 
frequencies of the customers by setting the length of the control interval and sizes of the 'behavior' databases.

\section{REFERENCES}

[1] Kilkki K. , Differentiated services for the Internet, Macmillan Technical Publishing, June 1999, Sections 2 and 3.

[2] Long-Tae Park; Jong-Wook Baek; Woon-Ki Hong, J., Management of service level agreements for multimedia Internet service using a utility model, IEEE Communications Magazine, Volume: 39 Issue: 5 , May 2001, Page(s): $100-106$.

[3] Dinesh Verma, Supporting Service Level Agreements on IP Networks, Macmillan Technical Publishing, 1999, Section 3.2.

[4] P. Bhoj, S. Singhal and S. Chutani, SLA management in federated environments, Computer Networks, Volume 35, Issue 1, January 2001, Pages: 5-24

[5] Internet2 - Qbone Bandwidth Broker, http://www.merit.edu/working.groups/i2-qbone-bb/

[6] G. R. Malan and F. Jahanian; An Extensible Probe Architecture for Network Protocol Performance Measurement, Proceedings of ACM SIGCOMM '98, September 1998

[7] Vern Paxson, End-to-End Internet Packet Dynamics, IEEE/ACM Transactions on Networking, Volume 7, No. 3, June 1999, Pages: 277-292.

[8] Bianchi, G.; Capone, A.; Petrioli, C., Throughput analysis of end-to-end measurement-based admission control in IP, Nineteenth Annual Joint Conference of the IEEE Computer and Communications Societies, INFOCOM 2000, Volume 3, 2000, Pages: 1461-1470

[9] Semret, N., Liao, R.R.-F., Campbell, A.T., Lazar, A.A., Pricing, provisioning and peering: dynamic markets for differentiated Internet services and implications for network interconnections, IEEE Journal on Selected Areas in Communications, Dec. 2000, Vol. 18, Issue 12, pp. 2499-2513

[10] J.-S. R. Jang, C.-T. Sun, E. Mizutani. Neuro-Fuzzy and Soft Computing - A Computational Approach to Learning and Machine Intelligence, Prentice Hall, Upper Saddle River, New Jersey,. 1997, Chapter 4.

[11] A. Pitsillides, Y. A. Sekercioglu, and G. Ramamurthy. Effective Control of Traffic Flow in ATM Networks Using Fuzzy Explicit Rate Marking (FERM) IEEE Journal on Selected Areas of Communications. Vol. 15, No. 2, February 1997, pp. 209 - 225.

[12] Rutong Zhang ; Jian Ma, Fuzzy QoS management in Diff-Serv networks; 2000 IEEE International Conference on Systems, Man and Cybernetics, 8-11 Oct. 2000, Vol 5, pp. 37523757.

[13] Yau-Hwang Kuo; Mong-Fong Horng; Jung-Hsien Chiang, An adaptive fuzzy traffic estimator of reservation protocols on IP-based networks, 1999 IEEE International Conference on Systems, Man, and Cybernetics, 1999. IEEE SMC '99, 12-15 Oct. 1999, Tokyo, Japan, vol.1, pp. 337 - 342

[14] WWW page of The Network Simulator - ns-2, http://www.isi.edu/nsnam/ns/ 